\title{
Development of “Kanako 2D (Ver.2.00)," a user-friendly one- and two-dimensional debris flow simulator equipped with a graphical user interface
}

\author{
Kana NAKATANI ${ }^{1}$, Takashi WADA ${ }^{1}$, Yoshifumi SATOFUKA ${ }^{2}$ and Takahisa MIZUYAMA ${ }^{1}$ \\ ${ }^{1}$ Department of Erosion Control Engineering, Kyoto University \\ (Oiwake-cho, Kitashirakawa, Sakyo-ku, Kyoto, Kyoto 6068502, Japan) \\ 2 Department of Civil Engineering, Ritsumeikan University (Noji-higashi 1-1-1, Kusatsu, Shiga 5258577, Japan)
}

\begin{abstract}
Debris flows often cause substantial losses of human life as well as economic losses. Damage can be estimated using numerical simulation models that describe the debris flow process. Some models can be used to determine the possible effects of sabo dams and have been practically employed to plan sabo dam arrangement. However, the existing simulation systems currently do not have efficient user interfaces, making it difficult for non-experts in debris flow simulations to run simulations without the aid of specialists. We developed a system that produces one- and two-dimensional debris flow simulations and is equipped with a graphical user interface. The system is based on an integration model and employs one-dimensional simulations of gully areas and two-dimensional simulations of alluvial fan areas; it then considers mutual influences in boundary areas between gullies and alluvial fans. Data can be input using a mouse and viewed on the monitor, and users can see real-time visualized images of a debris flow during a simulation. The interface enables users to run a debris-flow simulation without expert knowledge of the model, enabling better solutions for sabo engineering.
\end{abstract}

Keywords: Debris flow, graphical user interface, numerical simulation, one- and two-dimensional integration model.

\section{INTRODUCTION}

Japan has a mountainous topography, with approximately $70 \%$ of its area composed of steep areas under a weak geological condition. Thus, debris flows often result from heavy rains, causing substantial losses to human life as well as economic losses. Preventing and minimizing disasters caused by debris flows are critical to Japanese society and are among the most challenging problems facing the field of disaster prevention.

Sabo works can reduce and prevent sediment disasters such as debris flows. Sabo dams, or erosion and sediment control dams, effectively prevent and reduce disasters caused by debris flow, and many sabo dams have been constructed in Japan. These dams can reduce the energy of a debris flow, thereby controlling the surface erosion that usually occurs in upstream areas. For most efficient results, sabo dam design (e.g., type, location, size) must be customized to each specific area. Researchers have recently proposed a variety of numerical simulation models [Takahama et al.,
2002; Egashira and Itoh, 2004], and some have been put to practical use in sabo engineering.

However, many users have not been enthusiastic about the models because existing simulation systems are not equipped with efficient user interfaces. Simulation processes and displays are difficult to understand and require specific training to operate, and users find it difficult to prepare the various datasets and parameters. Thus, users who are not experts on debris flow simulation (e.g., sabo engineers) are unable to run simulations independently and are forced to rely on specialists.

As models become increasingly complex, the knowledge gap between specialists and engineers is widening, making simulations a complicated and impractical tool for sabo engineering. If researchers continue to focus on debris flow simulations by simply testing the accuracy of existing models or developing new models, the systems will continue to be impractical.

To address this problem, we developed a system equipped with a user-friendly graphical user interface (GUI), which will enable non-experts to 
run numerical debris flow simulations by themselves, without requiring expert knowledge of complicated parameters and models.

\section{REQUIREMENTS FOR A COMMONLY USED DEBRIS FLOW SIMULATION}

Making debris flow simulation a commonly used tool in sabo engineering requires a system equipped with a user-friendly GUI. A GUI-equipped system allows information visualization and helps non-expert users run numerical debris flow simulations easily [Takada and Koike, 2002; Iida et al., 2003]. Information visualization is a visual representation of abstract data that can reinforce human cognition and perception. It enables the viewer to gain knowledge about the internal structure of data and causal relationships. In a debris flow simulation, information visualization enables users to recognize debris flow simulation data such as discharge, flow depth, and sedimentation thickness as visual patterns and figures. It also allows users to browse many kinds of information simultaneously and to gain an instinctive understanding of data.

The system developed in this study incorporates information visualization. In addition, it permits users to simulate a mountainous river set with more than one sabo dam within the system. Users can also select different types of sabo dams, and the simulation can be run easily by simply modifying a few parameters. This permits an advanced examination of the sabo dam sediment control function or maintenance process.

\section{KANAKO VER. 2.00 SYSTEM}

\subsection{Platform}

We developed our system using MS Visual Basic.NET (VB.NET). We chose this platform because it was designed to run on Windows, provides an interactive GUI, and is easy for beginners to learn. There is a common misunderstanding that VB.NET is easy to use but has low performance. However, VB.NET is actually both easy to use and provides high performance. Further, because the platform is so widely used and easy to learn, users can easily modify and extend the system.

\subsection{System outline}

Using VB.NET, we developed a debris flow simulator equipped with a GUI. The system contains two parts: a user interface, which manages data

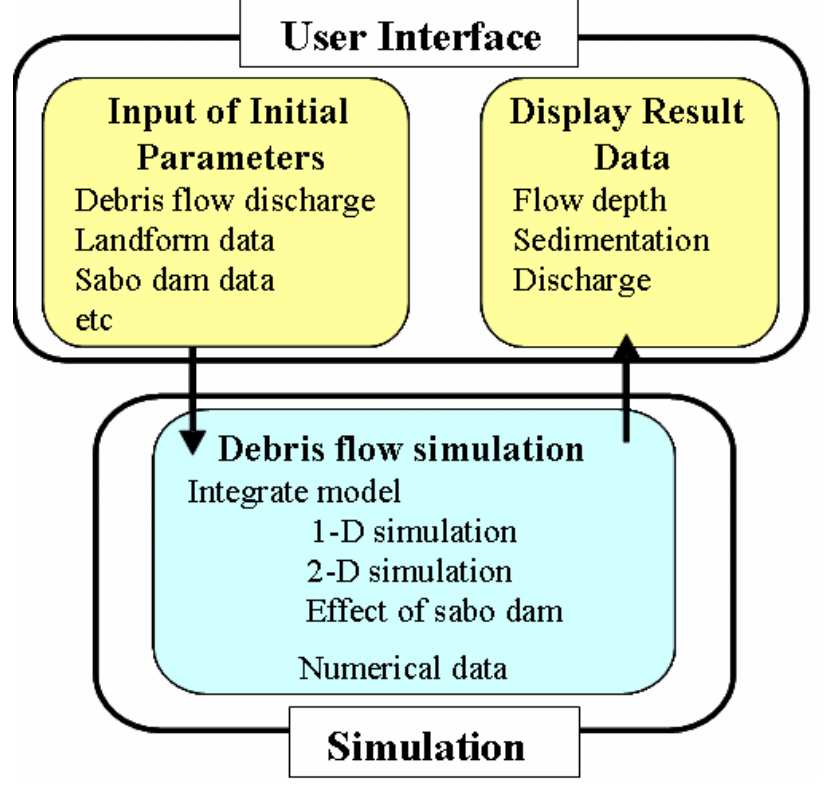

Fig. 1 Relation of the system's main functions.

input and display output; and a simulation, as shown in Figure 1.

The user interface permits users to input datasets for simulations using a mouse and to view them on a monitor; thus, the system is easy for beginners to use. During simulations, users can view real-time images of debris flows, hydrographs, and the effects of sabo dams.

The GUI-equipped debris flow simulator is called "Kanako." Kanako (Ver.1.10) [Nakatani et al., 2007] could simulate one-dimensional (1-D) debris flows and the effects of sabo dams. In this study, we developed Kanako 2D (Ver.2.00), which can simulate 1-D debris flow in gullies and two-dimensional (2-D) debris flow in alluvial fans. Table 1 shows the main functions of Kanako 2D (Ver.2.00)'s user interface.

Initial conditions are set through the user interface, after which the simulation begins; simulation results appear simultaneously through the user interface. The simulation part of the system employs the integration model [Wada et al., 2008], which is described in more detail in section 4 .

Kanako allows users who lack expert knowledge about debris flow numerical simulation to run simulations simply by setting some definite conditions. This is possible because the particularly complex parameters (e.g., Manning's roughness coefficient, coefficient of erosion or accumulation rate) and models have already been entered. Therefore, users can run the simulation without having to set or to use these parameters and models. Parameters can also be modified using numerical values from the default file. 
Table 1 Main functions in the Kanako 2D (Ver.2.00) user interface.

\begin{tabular}{|c|c|c|}
\hline & Function details & Explanation \\
\hline \multirow{8}{*}{ Input } & 1-D landform & Vertical section on steep gullies \\
\hline & 2-D landform & Plane figure on alluvial fans \\
\hline & Sabo dam & Type/height/location/number (1-D) \\
\hline & Hydrograph observation point & Number/location (1-D) \\
\hline & Supplied hydrograph & Input flow and concentration of debris flow \\
\hline & Initial movable bed layer & Thickness of movable layer before simulation \\
\hline & Field & Number of calculation points \\
\hline & Save/open data & Save/open all input data \\
\hline \multirow[t]{3}{*}{ Output } & \multirow[t]{2}{*}{$\begin{array}{c}\text { Display real-time animation during } \\
\text { simulation (simplified display) }\end{array}$} & $\begin{array}{l}\text { Display flow depth, sedimentation thickness } \\
\text { of initial bed on 1-D and 2-D landforms }\end{array}$ \\
\hline & & Display discharge at each observation point \\
\hline & Save result after simulation & Save detailed resulting data of the simulation \\
\hline
\end{tabular}

\subsection{User interface}

A user interface is an aggregate of means by which users interact with a particular machine, device, computer program, or system. In debris flow numerical simulation, user interface refers to the form of input and output data. For example, input involves setting initial conditions such as the landform condition and supplied debris flow discharge. The output describes simulation results such as discharge, flow depth, and sedimentation.

A GUI allows users to interact with media formats that employ graphic icons to represent the information and actions available to a user. Actions are usually performed through direct manipulation of the graphic elements.

The Kanako GUI system is easy for beginners to use because datasets used in simulations can be input using a mouse and viewed on a monitor. During simulations, users can view real-time images of debris flows, hydrographs, and the effects of sabo dams. The GUI enables users without specialized training to identify better solutions effectively and to run debris flow simulations independently.

\subsubsection{Data input/output through user interface}

When a user inputs information using a mouse, the user action is followed by a hidden process. For example, to set $z_{i}$, the $i$-th calculation point for riverbed altitude (the initial altitude setting is $30 \mathrm{~m}$, and the coordinate on the monitor is 300 pixels), the user points the mouse to the $z_{i}$ point on the screen, and $z_{i}$ is selected. The user drags $z_{i}$ to a certain position on the monitor (coordinate changes to 200 pixels) and then stops dragging (altitude is set at 20 $\mathrm{m})$. During this user operation, first, it is determined that point $z_{i}$ is being selected when the distance between the mouse pointer and $z_{i}$ is less than a certain value. Second, the positional coordinates of the mouse pointer can be acquired at any time while point $z_{i}$ is being dragged. Third, when dragging stops, the positional coordinates are recorded and the numerical value is converted into the data used in an actual simulation. Here, the new input condition of $z_{i}$ is set (see Fig. 2). A similar process is used to set sabo dam parameters such as dam height, type, and details of grid dam form on the "Set sabo dam details" screen. As the user clicks the "OK" button on the screen, the new parameters are recorded as new information in the system.

During the information output process, a real-time animation of debris flow is displayed. The system performs this action by continuously drawing the simulation result on the screen while the numerical simulation is running. The simulation interval $\Delta t$ is set as 0.01 second, as are the drawing intervals for riverbed deformation and flow depth represented by $1-\mathrm{D}$ and $2-\mathrm{D}$ landforms, and the discharge hydrograph of debris flow.

\section{NUMERICAL SIMULATION MODEL}

The system applies an integration model. For gullies, it uses a 1-D numerical simulation model to reproduce variations in a mountainous riverbed caused by debris flow and to simulate the effects of closed, slit, and grid types of sabo dams [Satofuka and Mizuyama, 2005]. For alluvial fans, it uses a 


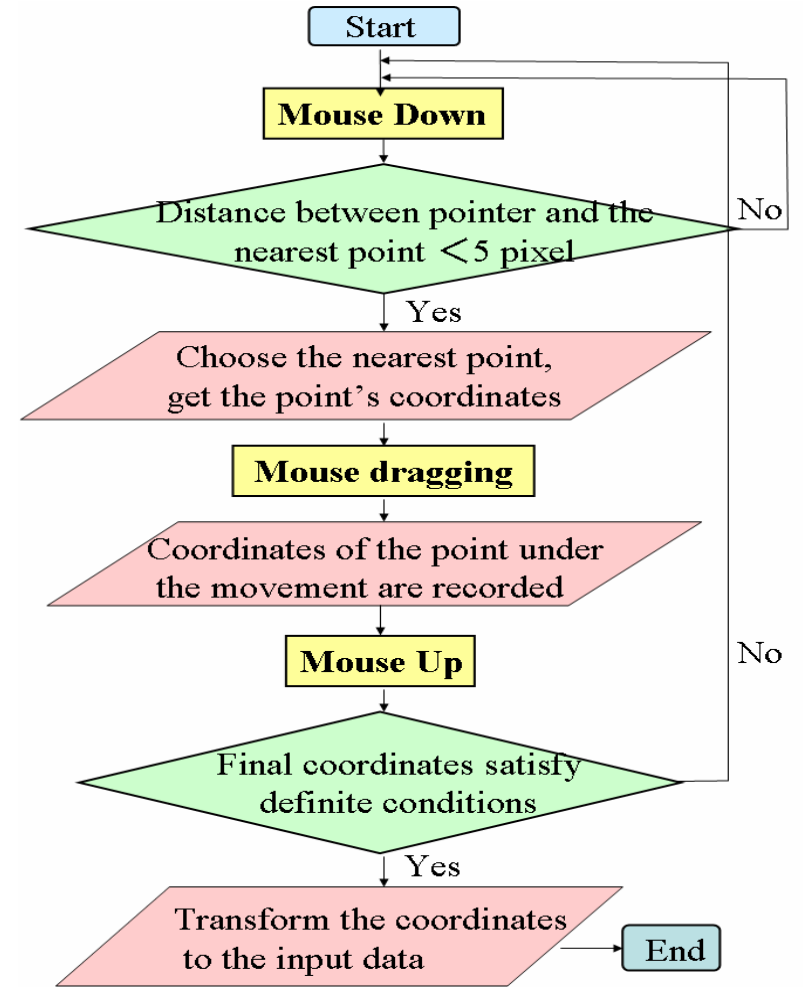

Fig. 2 Flow chart of data input during a mouse-dragging event in Kanako.

2-D numerical simulation model to reproduce changes in flow depth and sedimentation to simulate the passing area of debris flow.

For gullies, it uses a 1-D numerical simulation model to reproduce variations in a mountainous riverbed caused by debris flow and to simulate the effects of closed, slit, and grid types of sabo dams [Satofuka and Mizuyama, 2005]. For alluvial fans, it uses a 2-D numerical simulation model to reproduce changes in flow depth and sedimentation to simulate the passing area of debris flow.

Previous models have simulated the boundary areas between gullies and alluvial fans using the 1-D downstream end result as the 2-D upstream boundary condition. In contrast, our integration model simulates 1-D results for gullies and 2-D results for alluvial fans at each time step, incorporating mutual influences in both models. This enables the system to produce more accurate results, especially when marked sedimentation occurs in a boundary area.

\subsection{Governing equations for debris flow}

The basic 2-D debris flow equations are shown below. The system applies the same equations used in 1-D debris flow simulations, but includes $y$-axis direction terms. The equations for momentum, continuation, riverbed deformation, erosion/deposition, and riverbed shearing stress are based on previous research [Takahashi and Nakagawa, 1991], as are the staggered scheme and arrangement of variables [Takahashi and Kuang, 1987].

The continuation equation for the total volume of debris flow is

$$
\frac{\partial h}{\partial t}+\frac{\partial u h}{\partial x}+\frac{\partial v h}{\partial y}=i
$$

The continuation equation for determining the debris flow of the $k$-th grade of particle i

$$
\frac{\partial C_{k} h}{\partial t}+\frac{\partial C_{k} h u}{\partial x}+\frac{\partial C_{k} h v}{\partial y}=i_{k} C_{*}
$$

Here, two groups of grain-size classes are considered for sediment material: the larger grain size group, which affects grid-type sabo dam blockage; and the smaller grain size group, which does not affect blockage. When considering riverbed deformation (except when setting the upper point of a grid-type sabo dam), the average grain-size of all sediment materials is used, including both larger and smaller grain-size groups.

The phenomenon of $x$-axis direction (flow-) flow is given by a momentum equation, as follows:

$$
\frac{\partial u}{\partial t}+u \frac{\partial u}{\partial x}+v \frac{\partial u}{\partial y}=g \sin \theta_{w x}-\frac{\tau_{x}}{\rho h}
$$

The phenomenon of $y$-axis direction (cross-direction) flow uses a momentum equation, as follows:

$$
\frac{\partial v}{\partial t}+u \frac{\partial v}{\partial x}+v \frac{\partial v}{\partial y}=g \sin \theta_{w y}-\frac{\tau_{y}}{\rho h}
$$

The equation for determining change in bed surface elevation is as follows:

$$
\frac{\partial z}{\partial t}+i=0
$$

In Eq.(1)-(5), $h$ is flow depth, $u$ is $x$-axis direction flow velocity, $v$ is $y$-axis direction flow velocity, $C_{k}$ is the $k$-th sediment concentration by volume in debris flow, $z$ is bed elevation, $t$ is time, $i$ is erosion/deposition velocity, $i_{k}$ is the $k$-th sediment erosion/deposition velocity, $g$ is gravity acceleration, $\rho$ is interstitial fluid density, $\theta_{w x}$ and $\theta_{w y}$ are the flow surface gradients in the $x$-axis and $y$-axis directions, $C *$ is sediment concentration by volume in the movable bed layer, and $\tau_{x}$ and $\tau_{y}$ are the riverbed shearing stresses in the $x$-axis and $y$-axis directions, respectively.

\subsection{Conditions of simulation variables}

The numerical simulation model applies a staggered scheme using a finite difference method. Scalar and vector quantities are staggered by $\Delta x / 2$ 
(or $\Delta y / 2$ ) in the flow direction (or cross direction), as shown in Figure 3. Sabo dams are set at the calculation point of flow velocity, as shown in Figure 4. The effective flow depth, $h$ ' at the dam point, which is used to calculate the outflow flux, is expressed using the variables shown in Figure 4, as follows:

$$
h^{\prime}=\left\{\begin{array}{cc}
h_{i}+z_{i}-z_{d} & \left(h_{i}+z_{i}-z_{d} \geq 0\right) \\
0 & \left(h_{i}+z_{i}-z_{d}<0\right) \\
h_{i} & \left(z_{i}>z_{d}\right)
\end{array}\right.
$$

where $z_{d}$ is the dam crest elevation, which is the sum of bed elevation and dam height. Here, $h_{i}$ is flow depth in the scalar evaluation point next to the dam position in the upstream direction and $z_{i}$ is the riverbed height at the same point as $h_{i}$ (Fig. 4). In addition, at vector evaluation points where no sabo dam is set, flow discharge is calculated using the upstream side flow depth and velocity.

\subsection{Conditions of sabo dam design}

Kanako can simulate three types of sabo dam: closed, slit, and grid.

\subsubsection{Simulation of a closed-type sabo dam}

To examine upstream deposition velocity when a closed-type of sabo dam is set, a previously developed method [Takahashi et al., 2001] can be used to calculate variations in a riverbed. This method uses the relationship between dam height and riverbed height at the dam's upper site to determine whether materials will pass over a sabo dam. When a dam's upstream side contains a pocket, materials and water can be separated easily and immediately deposited.

\subsubsection{Simulation of a slit-type sabo dam}

Kanako can simulate the effect of a slit sabo dam. The system simulates the narrowing river

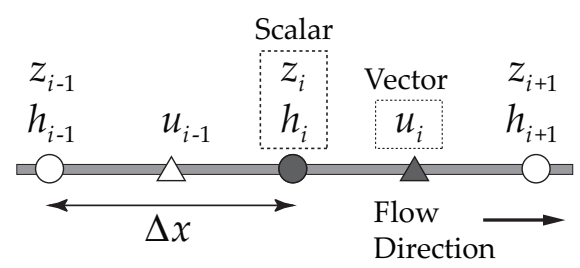

Fig. 3 Arrangement of variables in a 1-dimensional area.

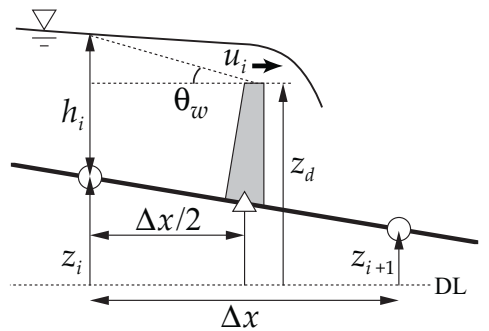

Fig. 4 Arrangement of variables at the sabo dam. width between upstream and downstream scalar evaluation points, from the vector evaluation point where the slit-type of sabo dam is set. However, the model is not able to incorporate energy loss from the sharp narrowing of river width.

\subsubsection{Simulation of a grid-type sabo dam}

When debris flows occur in a mountainous river where a grid type of sabo dam is set, the opening of the grid dam is blocked by large rocks in the front part of the debris flow, which can cause the dam to trap the subsequent muddy flow in the same way as a closed-type dam. A previously developed model [Satofuka and Mizuyama, 2006] treats a grid dam as a closed dam with a temporally variable height through the blockage; sediment material is composed of two groups of grain-size classes, but only the larger group class of sediment causes stoppage of the grid dam opening and increases dam height.

The growth rate of dam height $i_{d}$ ", which includes the influence of a horizontal beam, can be expressed using Eq.(7). Deposition caused by blockage between the columns is assumed to be independent from the deposition caused by the influence of horizontal beams:

$$
i_{d}{ }^{\prime \prime}=a_{2} \frac{C}{C_{*}} \frac{h}{d} v
$$

where $d$ is boulder diameter and $z_{L}^{j}$ and $z_{H}^{j}$ are the elevations of the undersurface and the upper surface of the $j$-th beam, respectively. In cases when the bed is between the $j$-th beam and the $j+1$-th beam, the beam influence changes according to whether the gap between the undersurface of the $j+1$-th beam and the bed is larger or smaller than the boulder's diameter. If the gap under the beam is smaller than the boulder's diameter, a fraction of debris flow is deposited from the bed to the upper surface of the beam. In this kind of situation, $a_{2}$ in Eq. (7) is calculated as

$$
a_{2}=a_{1}+\left(z_{H}^{j+1}-z_{d}\right) / h
$$

where $z_{d}$ is bed elevation immediately upstream of the dam, and $z_{L}^{j}$ and $z_{H}^{j}$ are elevations of the undersurface and the upper surface of the $j$-th beam, respectively.

In contrast, if the gap under the beam is larger than the boulder's diameter, the beam's influence can be ignored. In this case, $a_{2}$ is calculated as

$$
a_{2}=a_{1}
$$

where $a_{1}$ is an approximation using parameters such as boulder concentration and opening width.

Since Eq.(7) calculates the rate of increase in bed elevation in the area directly upstream of the 
dam with longitudinal length $d$, this equation cannot be used directly to calculate variation in the riverbed. It can be modified by using the interval of calculating point $\Delta x$ united with the usual deposition velocity $i_{b}$, which is determined by the imbalance between a flow and riverbed slope as follows:

$$
i_{b}{ }^{\prime}=i_{b}-i_{d}{ }^{\prime \prime} \frac{d}{\Delta x}
$$

Erosion and deposition velocity values, commonly used in these simulations, are positive for erosion and negative for deposition. Therefore, $i_{d}$ ", is negative in Eq. (10) because deposition is positive in the calculation of $i_{d}$ '”.

The growth rate of dam height can be calculated by substituting large particle concentration, flow velocity, and flow depth at the dam point in Eq.(7). This rate can be changed into erosion/deposition velocity using Eq.(10) and then used to calculate variation in the riverbed.

\subsection{Integration model}

\subsubsection{Outline of the integration model}

Figure 5 presents an outline of the integration model, which enables continual simulation of both a 1-D modeling of gullies and 2-D modeling of alluvial fans at each time step. The integration model uses an explicit method.

At time $t$, the calculated 1-D downstream end-flow discharge and sediment discharge are applied as the 2-D upstream inflow condition, and 2-D upstream riverbed height and flow depth are calculated using the forward condition. Then at time $t+\Delta t$, the calculated riverbed height and flow depth value are applied as the 1-D downstream end

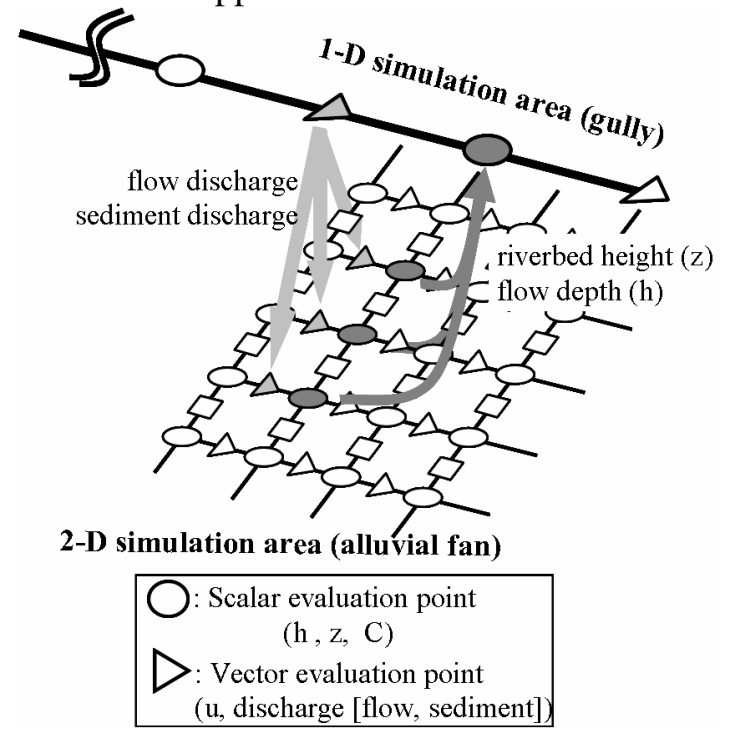

Fig. 5 Outline of the integration model. boundary condition. Therefore, the integration model incorporates mutual influences of the 1-D and 2-D simulations. Moreover, this integration model can handle situations in which intervals of 2-D cross-direction calculation points differ from 1-D river width, or in which 1-D simulation flow direction differs slightly from 2-D simulation flow ( $x$-axis) direction.

\subsubsection{Integration of 1-D and 2-D simulation areas}

The 1-D downstream end calculation point number is set as ie, and the inflow point in the 2-D upstream end area's grid point coordinates are set as $(1, j c)$. Figure 6 shows the integration of $1-\mathrm{D}$ and 2-D areas, corresponding to the 1-D no. (ie-1) vector evaluation point with the 2-D $(1, j c)$ vector evaluation point. In the figure, $\Delta x_{1}$ shows the calculation point interval in $1-\mathrm{D}, \Delta x_{2}$ shows the $x$-axis direction grid interval in 2-D, $h_{i}$ and $z_{i}$ represent the flow depth and riverbed elevation of the 1-D area no. $i$ point, $h$ ' and $z$ ' represent the average flow depth and riverbed elevation of the 2-D area upstream end, respectively, and $\theta_{t}$ shows the angle between the 1-D $x$-axis and the 2-D $x$-axis. To integrate different dimensional simulation areas, $\theta_{I}$ must be considered.

Figure 6 also shows that the 2-D grid interval in the $x$-axis direction is transformed to $\Delta x_{2} \cos \theta_{i}$ in the 1-D area, and the 1-D no. (ie-1) vector evaluation point river width is shown as $B_{i e-1} / \cos \theta_{i}$ in the 2-D area. In Figure 7, $B_{i e-1}$ shows the river width at the 1-D no. (ie-1) vector evaluation point, and $\Delta y_{2}$ shows the 2-D grid interval in the $y$-axis direction.

\subsubsection{2-D simulation area inflow condition}

Considering the 2-D simulation area inflow condition, we can divide $Q_{i e-1}$, which is the discharge of the 1-D no. (ie-1) vector evaluation point, to obtain 2-D area inflow points. Discharge $Q_{i e-1}$ is represented as the product of flow velocity, flow depth, and river width, shown as Eq.(11):

$$
Q_{i e-1}=u_{i e-1} h_{i e-1} B_{i e-1}
$$

where $u_{i e-1}$ is the flow velocity of the 1-D no. (ie-1)

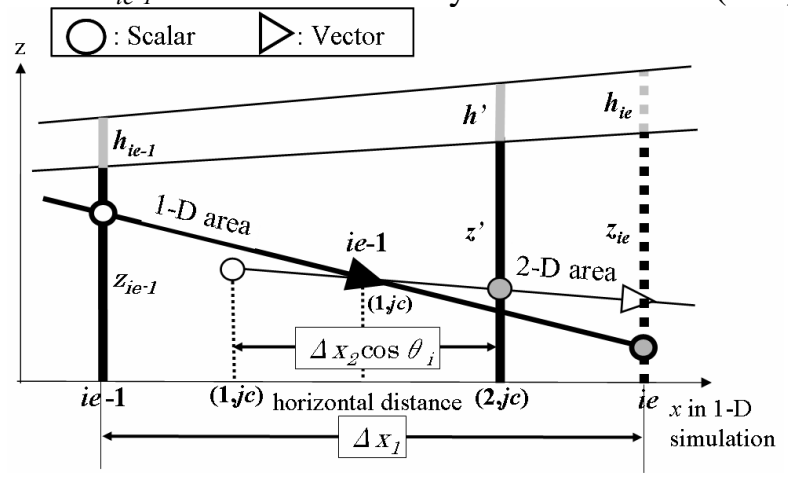

Fig. 6 Profile of the integration area. 
vector evaluation point.

First, we divide $Q_{i e-1}$ into two elements: $x$-direction and $y$-direction on the 2-D simulation area, and $x$ - and $y$ - directions at right angles. As shown in Figure 8, $Q_{x}$ and $Q_{y}$ are the divided $x$ - and $y$-direction discharge, described by Eq.(12) and (13):

$$
\begin{aligned}
& Q_{x}=u_{i e-1} \cos \theta_{i} \cdot h_{i e-1} \cdot B_{i e-1}\left|\cos \theta_{i}\right| \\
& Q_{y}=-u_{i e-1} \sin \theta_{i} \cdot h_{i e-1} \cdot B_{i e-1}\left|\sin \theta_{i}\right|
\end{aligned}
$$

Next, we can divide and supply $Q_{x}$ and $Q_{y}$ to the 2-D simulation area inflow points. More than one inflow point can be used in a 2-D simulation area; the number changes according to $B_{i e-1} / \cos \theta_{i}$. As shown in Figure 9, inflow points are expanded to the 2-D simulation area's $y$-direction, where the 2 -D $(1, j c)$ vector evaluation point is set at the center.

The number of inflow points in the 2-D simulation area is always set at an odd number as shown in Eq.(14). Here, function $\operatorname{int}(x)$ shows the integer of number $x$ :

$$
N=2 \times \operatorname{int}\left(\frac{B_{i e-1} / \cos \theta_{i}}{2 \Delta y_{2}}+\frac{1}{2}\right)+1
$$

When $N \geq 3$, the number of $x$-direction main inflow points is set as $(N-2)$, the $x$-direction end inflow point is set as 2 , and the $y$-direction inflow point is set as $(N-1)$. The supplied discharge of each inflow point is described below.

Discharge of the $x$-direction main inflow point:

$$
Q_{x(1, j)}^{\prime}=Q_{x} \frac{\Delta y_{2}}{B_{i e-1} / \cos \theta_{i}}
$$

$$
(j c-(N-3) / 2 \quad j \quad j c+(N-3) / 2)
$$

Discharge of the $x$-direction end inflow point:

$$
\begin{gathered}
Q_{x(1, j)}^{\prime}=Q_{x} \frac{B_{s}}{B_{i e-1} / \cos \theta_{i}} \\
(j=j c-(N-1) / 2, j c+(N-1) / 2)
\end{gathered}
$$

Discharge of the $y$-direction inflow point:

$$
\begin{gathered}
Q_{y(2, j)}^{\prime}=\frac{Q_{y}}{N-1} \\
(j c-(N-1) / 2 j \quad j c+(N-3) / 2)
\end{gathered}
$$

In the above equations, $Q_{x(i, j)}^{\prime}$ and $Q_{y(i, j)}^{\prime}$ are the $x$-axis and $y$-axis discharge of 2-D $(i, j)$, and $B_{S}$ is one-half of the remainder dividing $\left(B_{i e-1} / \cos \theta_{i}\right)$ by $\Delta y_{2}$.

When $N=1$, we can set the first $x$-direction main inflow point at 2-D $(1, j c)$ and the second $y$-direction inflow point at 2-D $(2, j c-1),(2, j c)$. The supplied discharge of each inflow point is described

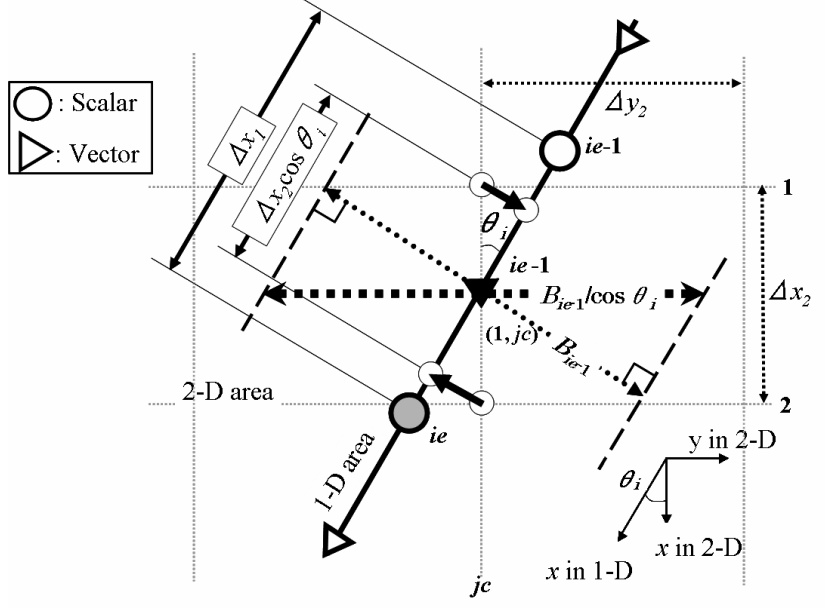

Fig. 7 Plan of the integration area.

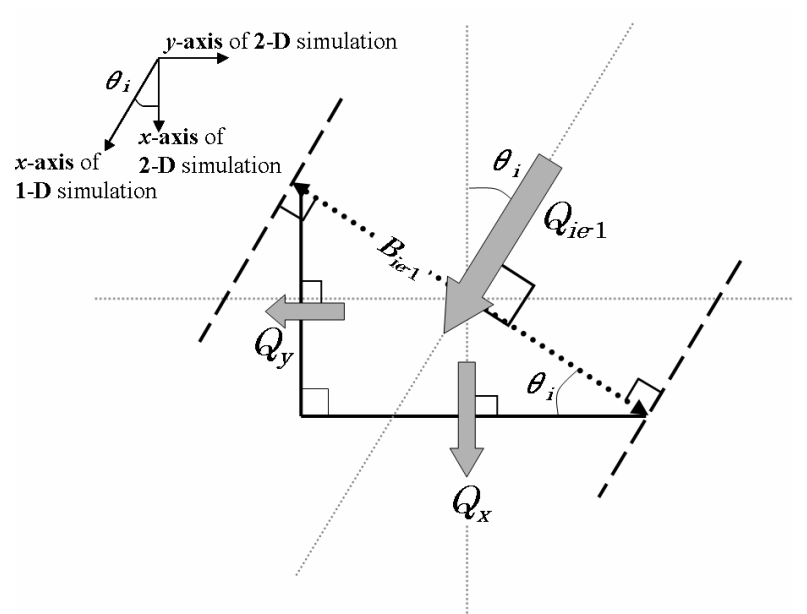

Fig. 8 Division of the flux $Q_{i e-1}$ at no. (ie-1) calculation point for vector volumes.

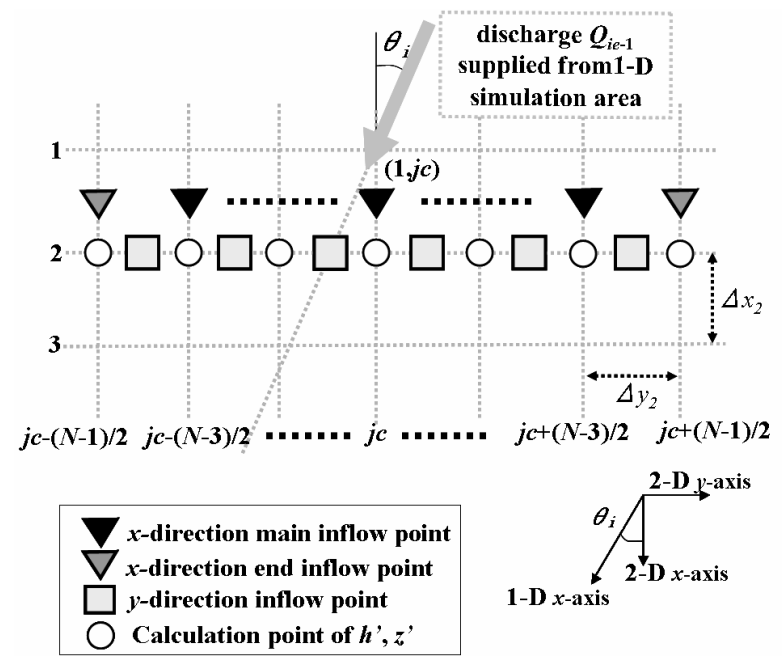

Fig. 9 Inflow condition at the upstream border of the two-dimensional calculation area.

below.

Discharge of the $x$-direction main inflow point:

$$
Q_{x(1, j c)}^{\prime}=Q_{x}
$$


Discharge of the $y$-direction inflow point:

$$
Q_{y(2, j)}^{\prime}=\frac{Q_{y}}{2} \quad(j=j c-1, j c)
$$

Supplied sediment discharge is calculated by multiplying the inflow discharge and $C_{i e-1}$, where $C_{i e-1}$ shows the 1-D no. (ie-1) scalar evaluation point sediment concentration.

\subsubsection{Boundary condition of the 1-D simulation area downstream end}

We can set the 1-D downstream end boundary condition by calculating $z_{i e}$ and $h_{i e}$ from $z_{i e-1}, h_{i e-1}, z$, and $h^{\prime}$, where $z^{\prime}$ and $h^{\prime}$ are the average of riverbed altitude and flow depth, shown as an open circle $\bigcirc$ in Figure 9. At each point, we can adaptively weight the contribution rate to $1-\mathrm{D}$ river width and sum all the values; $z$ ' is calculated using Eq.(20) and Eq.(21), and $h$ ' is calculated similarly:

$N \geq 3$

$$
\begin{aligned}
& z^{\prime}=\sum_{j=j c-\left(\frac{N-3}{2}\right)}^{j c+\left(\frac{N-3}{2}\right)} z(2, j) \times \frac{\Delta y_{2}}{B_{i e-1} / \cos \theta_{i}}+ \\
& \left\{z\left(2, j c-\frac{N-1}{2}\right)+z\left(2, j c+\frac{N-1}{2}\right)\right\} \times \frac{B_{s}}{B_{i e-1} / \cos \theta_{i}}
\end{aligned}
$$

$N=1$

$$
z^{\prime}=z(2, j c)
$$

As shown in Figure 6, $z_{i e}$ and $h_{i e}$ are calculated by extrapolating $z_{i e-11}, h_{i e-1}$, and $z^{\prime}, h^{\prime} ; z_{i e}$ is calculated using Eq.(22), and $h_{i e}$ is calculated similarly:

$$
z_{i e}=\frac{1}{\Delta x_{1}+\Delta x_{2} \cos \theta_{i}} \times\left\{\left(\Delta x_{2} \cos \theta_{i}-\Delta x_{1}\right) z_{i e-1}+2 \Delta x_{1} z^{\prime}\right\}
$$

\section{OUTLINE OF KANAKO 2D (VER. 2.00)}

\subsection{Landform settings}

A longitudinal figure representing the 1-D riverbed profile appears in the upper part of the Kanako start screen (Fig. 10), and a plane figure displaying the 1-D river width (presented in meters) and 2-D landform plane appears in the middle part of the screen. The 1-D and 2-D areas are placed successively. The 1-D riverbed profile includes three parameters: the surface of the moveable bed, the surface of the fixed bed, and the river width. Each can be modified by dragging the corresponding point. Yellow points on the riverbed profile indicate hydrograph observation points, and black, gray, and blue checked rectangles indicate closed-, slit-, and grid-type sabo dams, respectively. A guide appears when a point is dragged, revealing the current position.

To set 2-D landform conditions, users open the 'set 2-D landform' window (Fig. 11) and use the box to set parameters: the slope of the landform, angle to the river center axis, and center angle of the alluvial fan, shown as $\theta_{1}, \theta_{2}$, and $\theta_{3}$, respectively, in Figure 11.

In both 1-D and 2-D landform settings, users can set detailed data using numerical values in the numerical setting screen (Fig. 12). In addition, if a dataset is saved in the designated format for Kanako, users can import an external data file to set landform parameters.

\subsection{Supplied hydrograph settings}

To modify the supplied hydrograph from the upstream end, users can set data in the 'Set supplied hydrograph' screen, shown in Figure 13. The discharge of debris flow can be modified by dragging the red points up and down. While dragging, two numbers appear (see A in Fig. 13), representing time and discharge of debris flow,

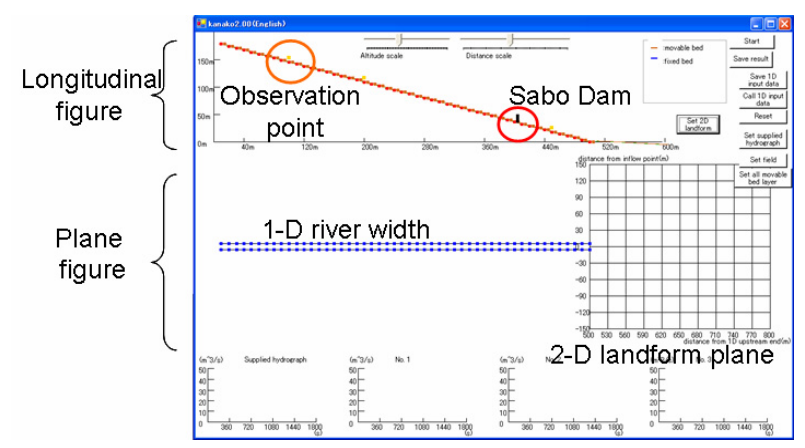

Fig. 10 Start screen (one-dimensional landform setting).

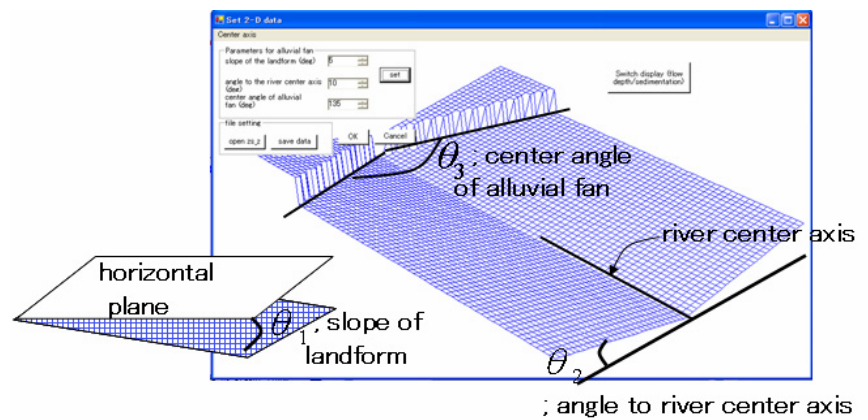

Fig. 11 Two-dimensional landform setting screen.

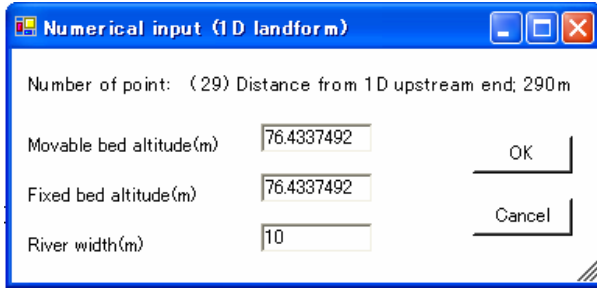

Fig. 12 Numerical input screen. 
respectively. The supplied sediment concentrations can be modified by changing the numbers in these text boxes. During the simulation, a real-time hydrograph animation is displayed at the left end of the graph under 'supplied hydrograph.'

\subsection{Sabo dam details setting}

Sabo dam parameters can be set in the sabo dam detail screen (Fig. 14). Users double-click on the sabo dam, which causes a pop-up window of detailed dam settings to appear. Variable settings include height, type (closed, slit, or grid), and slit width, which can be modified using text boxes or radio buttons. Dam position can also be adjusted by dragging the rectangle right or left along the river profile.

Users can enter other details for a grid type of sabo dam, such as column diameter and distance between columns, using a different screen (Fig. 15). They can set grid dam details using the spin control or by directly inputting numerical values.

Most existing grid-type sabo dams have a fixed grid diameter and distance between each grid. However, recent research [Mizuno and Mizuyama, 1996] has indicated that grid dams designed to have narrow pipe intervals in upper grids are capable of trapping a greater volume of sediment transported by debris flow. Therefore, in the near future, most grid-type sabo dams will have a thinner diameter and shorter distance between horizontal beams in the upper part.

Kanako allows horizontal beam diameter and distance to be set individually.

\subsection{Running a simulation}

The simulation starts when a user clicks the 'Start' button. Two screens are then displayed: the main screen and the 2-D landform screen. First, debris flow is initiated and moves down from the upper stream end. The upper area of the main screen (Fig. 16) presents an animated real-time image of the flow depth, moving bed surface, initial bed surface, and fixed bed in the longitudinal figure. The middle area of the screen shows flow depth and sedimentation in the plane figure.

The bottom of the main screen has four hydrograph and sediment graphs, supplied from data at the upstream end and at each observation point. The vertical axis represents debris flow discharge $\left(\mathrm{m}^{3} / \mathrm{s}\right)$ and the horizontal axis represents time $(\mathrm{s})$.

After debris flow reaches the alluvial fan, the 2-D landform screen (Fig. 17) presents an animated image of flow depth and sedimentation on the set landform, and represents the passing area of debris flow.

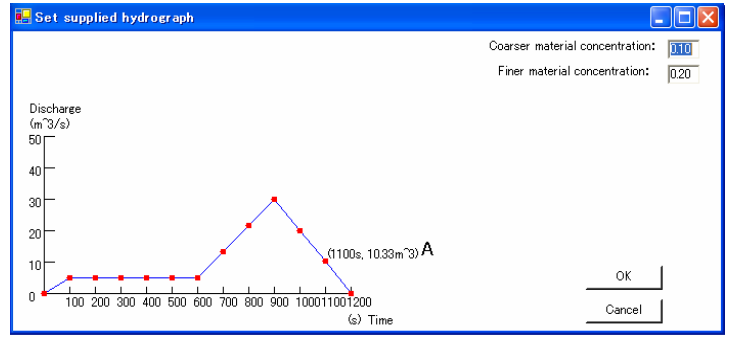

Fig. 13 Setting the supplied hydrograph screen.

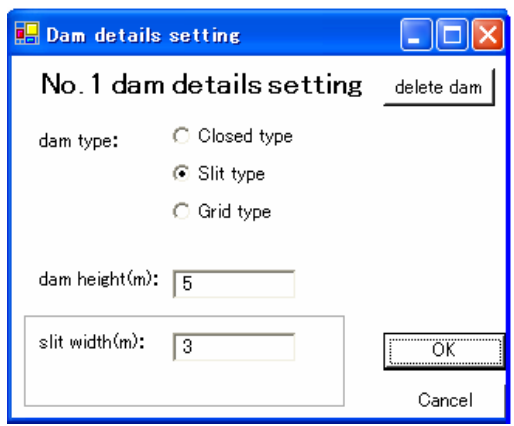

Fig. 14 Sabo dam detail setting screen.

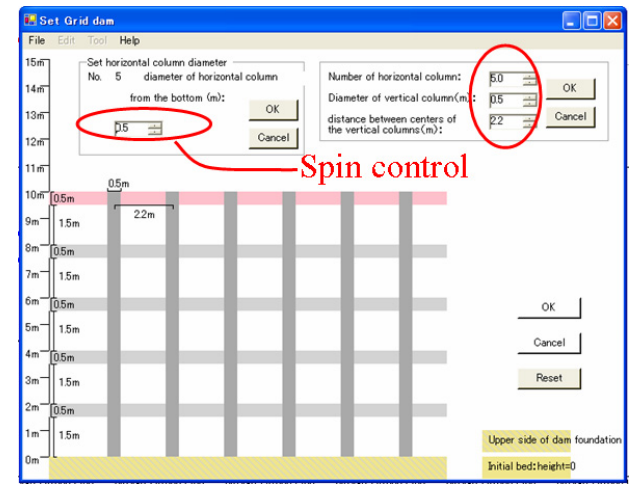

Fig. 15 Grid-type sabo dam details setting screen.

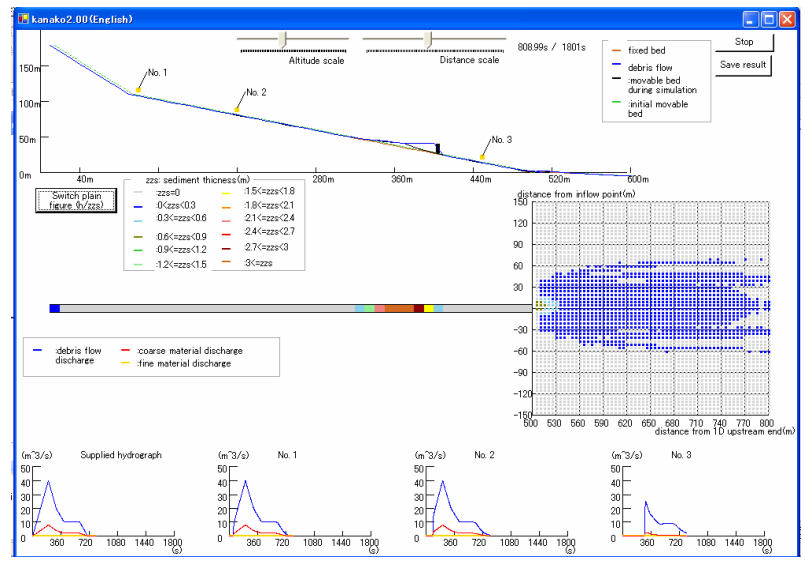

Fig. 16 Simulation screen (main screen, plane figure showing sedimentation thickness).

The simulator calculates for a period of $1800 \mathrm{~s}$ and stops; this is the initial setting for simulation continuance time, but it can be modified by users. After running a simulation, users can save simulation results as a data file following normal 


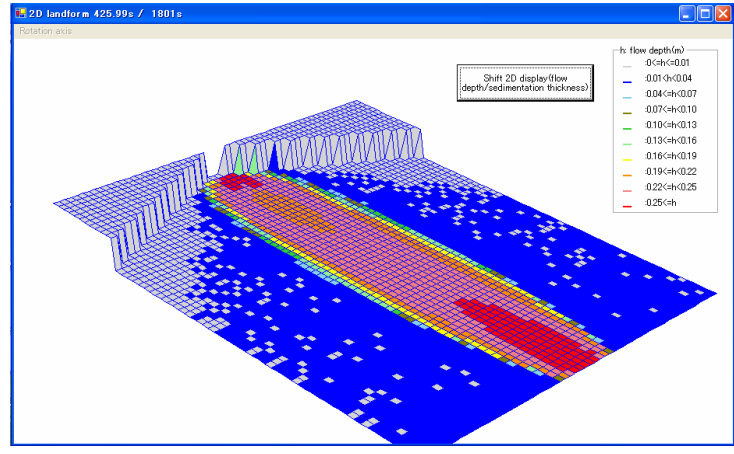

Fig. 17 Two-dimensional simulation screen (showing flow depth).

procedures for saving data.

\section{CONCLUSIONS AND FUTURE RESEARCH}

The GUI-equipped debris flow simulator Kanako 2D (Ver.2.00) is easier to use and produces more information than previously developed simulators. Users can easily input calculation conditions based on their needs, by using a mouse to click on the menu functions and viewing data on the monitor. Simulation results are displayed graphically, enabling an intuitive understanding. Therefore, users can conduct numerical simulations to calculate debris flow processes and sabo dam effects even without advanced training. In addition, by simply modifying a few parameters Kanako can easily simulate various types of sabo dam settings, including different types, locations, sizes, and combinations when a simulation involves more than one sabo dam. This enables advanced investigation of sabo dam sediment control functions and maintenance processes.

Kanako (Ver.1.02) can provide a 1-D simulation of a mountainous river with a closed-type or slit-type sabo dam. The Japanese version can be downloaded at no cost from the "SABO Technical Center (STC)" website:

http://www.stc.or.jp/index.html

Kanako (Ver.1.10) also provides a 1-D simulation of a mountainous river with a grid-type sabo dam. The English version can be downloaded free of charge from the "The Online Library of Civil and Environmental Engineering" website:

http://www.olcivil.com/Site/index.php

In future research, we plan to incorporate new advances in debris flow simulation modeling to the simulation part of the Kanako system. For example, researchers are now proposing a new type of sabo dam that is equipped with shutters and are focusing more on the ecosystems and sediment runoff in mountainous rivers. If new types of sabo dams are successful, it will be important to include their sediment control mechanisms into the simulation part of the Kanako system.

We also plan to improve the user interface part of the Kanako system by incorporating a geographic information system (GIS) function into the 2-D landform input function, making input operation even easier. In addition, the current Kanako system has many more functions than the original Ver.1.00, so the main screen has many more buttons. Future research may result in even more functions, which will make the system more complex and difficult to understand. This is contrary to our original intention, which was to develop a simple, effective, user-friendly system. To solve this problem, we plan to develop a semi-customized system, in which each user can set the main screen functions depending on their needs. This should enable the system to maintain a high-quality simulation of debris flow but remain user-friendly.

No matter how user-friendly a system is, non-expert users will still find it difficult to determine independently the ideal type, number, size, location, and combination of sabo dams to determine the ideal "sabo solution."

Therefore, a new-generation algorithm is required to determine automatically the best sabo solution under given conditions. This new algorithm must incorporate the scale of damage caused by debris flow, considering the discharge of debris flow, discharge of material, transferred discharge of material, size of material, and the degree to which the disaster will affect human life and the economy. By developing this algorithm and incorporating it into the Kanako system, both expert and non-expert users should be able to conduct sophisticated sabo planning.

\section{REFERENCES}

Egashira, S., Itoh, T. (2004), Numerical simulation of debris flow, Journal of Japan Society of Computational Fluid Dynamics, $12(2)$ www.nagare.or.jp/jscfd/j-jscfd/122/p122-2.pdf, accessed on 2008.6.1(in Japanese).

Iida, S., Igarashi, T., Takaki, S. (2003), A study of flood emergency management action support system which applies distributed simulation integration technology, Journal of River Engineering, JSCE, Vol. 9, pp. 161-166 (in Japanese with English abstract).

Mizuno, H., Mizuyama, T. (1996), Experimental study of a grid dam with narrow pipe interval of upper grids, Journal of the Japan Society of Erosion Control Engineering, Vol. 49, No. 4, pp. 3-8, (in Japanese with English abstract).

Nakatani, K., Satofuka, Y., Mizuyama, T.(2007), Development of 'KANAKO', a wide use debris flow simulator equipped with GUI, Proc. of 32nd Congress of IAHR, Venice, Italy, 
CD-ROM, 10 p, A2.c-182.

Satofuka, Y., Mizuyama T. (2005), Numerical simulation of a debris flow in a mountainous river with a sabo dam, Journal of the Japan Society of Erosion Control Engineering, Vol. 58, No. 1, pp. 14-19, (in Japanese with English abstract).

Satofuka, Y., Mizuyama, T. (2006), Numerical simulation of debris flow control by a grid dam, Proc. of the 6th Japan-Taiwan Joint Seminar on Natural Hazard Mitigation, CD-ROM.

Takada, T., Koike, H. (2002), MieLog: A highly interactive visual $\log$ browser using information visualization and statistical analysis, Proceedings of LISA XVI Sixteenth Systems Administration Conference, The USENIX Association, pp. 133-144.

Takahama, J., Fujita, Y., Kondo, Y., Hachiya, K. (2002), Two-layer model for analysis of deposition and erosion processes of debris flows, Annual Journal of Hydraulic Engineering, JSCE, Vol. 46, pp. 677-682 (in Japanese with
English abstract).

Takahashi, T., Kuang, S. (1986), Formation of debris flow on varied slope bed, Disaster Prevention Research Institute Annuals, 29(B-2), pp. 345-349.

Takahashi, T., Nakagawa, H. (1991), Prediction of stony debris flow induced by severe rainfall, Journal of the Japan Society of Erosion Control Engineering, Vol. 44, No. 3, pp. 12-19 (in Japanese with English abstract).

Takahashi, T., Nakagawa, H., Satofuka, Y., Kawaike, K. (2001), Flood and sediment disasters triggered by 1999 rainfall in Venezuela: A river restoration plan for an alluvial fan, Journal of Natural Disaster Science, 23, pp. 65-82.

Wada, T., Satofuka, Y., Mizuyama T. (2008), Integration of 1and 2-dimensional models for debris flow simulation, Journal of the Japan Society of Erosion Control Engineering, Vol. 61, No. 2, pp. 36-40 (in Japanese with English abstract). 